\title{
La consolidación de la educación pública brasileña en el estado de Mato Grosso (1889-1908) ${ }^{1}$
}

\author{
Consolidation of Brazilian public education in \\ the state of Mato Grosso (1889-1908)
}

\author{
A consolidação da educação pública brasileira no \\ estado de Mato Grosso (1889-1908)
}

\section{Newenkonchi Brasil komgen kimeltuwün zugu feyti \\ Mato Grosso xokiñ mapu mew (1889-1908)}

\author{
Nilce Vieira Campos Ferreira ${ }^{2}$ \\ Universidade Federal do Mato, Brasil \\ Yésica Paola Montes Geles ${ }^{3}$ \\ Universidad Pontificia Bolivariana, Colombia
}

Recepción: 10/08/2018

Evaluación: 21/05/2019

Aceptación: 23/05/2019

Artículo de Investigação

DOI: https://doi.org/10.19053/01227238.9804

El presente artículo tiene como ob- responsables de la educación pública en jetivo examinar el fortalecimiento de la educación pública mato-grossense en el periodo de 1889-1908, a través del análisis de las legislaciones gubernamentales descritas en los informes emitidos por los dicho tiempo. Para ello, se plantearon dos preguntas base que orientaron el análisis: ¿hubo consonancia entre la defensa de la escuela pública moderna y el modelo de escuela establecida en Cuiabá, capital del

1 Este texto surge de una investigación desarrollada dentro de un proyecto de investigación más amplio con una duración 2014-2015, n. ${ }^{\circ}$ de registro 060 / CAP / 2014 / UFMT / Cuiabá y la intención de crear una imagen de la instrucción pública en el estado de Mato Grosso (Brasil) en la época republicana. Actualmente está vinculada a la investigación que tiene el apoyo del Consejo Nacional de Desarrollo Científico y Tecnológico (CNPq) y está inscrita en el marco del macro proyecto "Formación de Profesoras Misioneras en las Regiones Centro-Oeste y Norte: Mato Grosso y Rondonia/Brasil (1936-963)", coordinado por Nilce Vieira Campos Ferreira y propuesto para ser ejecutado entre 2018-2021.

2 Doctora en Educación. Profesora del Instituto de Educación e del Programa de Pos-Graduación en Educación, Universidade Federal do Mato - Campus Cuiabá/MT (UFTM/IE/PPGE/DTFE). Grupo de investigación: - Grupo de Pesquisa e Estudos em História da Educação, Instituições e Relações de Gênero-GPHEG. Rede de Pesquisa, Ensino e Extensão em Educação nas regiões Centro-Oeste e Norte do Brasil e na América Latina - RECONAL-Edu, correo eletrónico: nilcevieiraufmt@gmail.com.

3 Magíster en Educación de la Universidade Federal de Mato Grosso - Campus Cuiabá/MT (UFTM/IE/PPGE/DTFE). Docente adscrita al Centro de Lenguas de la Universidad Pontifícia Bolivariana, campus Montería. Grupo de investigación: Grupo de Pesquisa e Estudos em História da Educação, Instituições e Relações de Gênero-GPHEG. correo eletrónico: yesica.montes.g55@gmail.com. 
estado de Mato Grosso?; y ¿las escuelas públicas cuiabanas fueron subsidiadas con los materiales y la infraestructura adecuados para el desarrollo de actividades en el aula? Se utilizaron, como origen de la información, las fuentes históricas oficiales, tanto como los mensajes de los presidentes estatales, las leyes o los decretos relacionados con la educación pública primaria y los informes de la educación pública desde 1889 hasta 1908. Gracias al estudio se concluye que, en 1889 , año de la proclamación de la República de Brasil, persistían los problemas existentes en las escuelas de Mato Grosso como la precariedad y la falta de recursos para la enseñanza, así como falta de profesionales cualificados, temas que fueron expuestos repetidamente en los mensajes emitidos por el gobierno.

Palabras clave: Revista historia de la educación latinoamericana; descentralización de la educación; instrucción pública; historia de la educación en Mato Grosso.

\section{ABSTRACT}

The aim of this article is to examine the strengthening of public education of Mato Grosso between 1889-1908, through the analysis of government legislations described in the reports issued by those who were in charge of public education at that time. For this purpose, two leading questions were posed to guide the analysis: was there agreement between the defense of the modern public school and the school model established in Cuiabá, capital of the state of Mato Grosso?; and were the public schools in Cuiabá subsidized with adequate materials and infrastructure for the development of classroom activities?
Both official historical sources and messages from state presidents, laws or decrees related to public primary education, and public education reports from 1889 to 1908 were used for this study. The study concluded that, in 1889, when the proclamation of the Republic of Brazil took place, problems such as precariousness and lack of resources for teaching, as well as lack of qualified professionals, persisted in schools in Mato Grosso. These obstacles were repeatedly exposed in the messages issued by the government.

Keywords: Journal History of Latin American Education; decentralization of education; public instruction; History of education in Mato Grosso.

\section{RESUMO}

Este artigo tem como objetivo discutir a consolidação da educação pública mato-grossense no período de 1889-1908, por meio da análise das atividades educativas propostas nas legislações governamentais e descritas nos relatórios emitidos pelos responsáveis pela instrução pública mato-grossense no período em estudo. Trazemos duas perguntas que orientaram a análise: ¿A defesa da escola pública moderna era coetânea e coerente com o modelo de escola estabelecido em Cuiabá? ¿Escolas públicas cuiabanas foram subsidiadas com recursos pedagógicos e infraestrutura adequada para o desenvolvimento do ensino nas salas de aula?
Como fontes de informação, utilizamos fontes históricas oficiais como mensagens dos presidentes do estado de Mato Grosso, leis e decretos presidenciais, bem como os relatórios de Instrução Pública desde 1889 a 1908. Após nossas análises, concluímos que entre os anos de 1889, ano da proclamação da República de Brasil, até os anos de 1908, persistiam os problemas existentes nas escolas de Mato Grosso, a exemplo, a precariedade e a falta de recursos financeiros, e pedagógicos para o ensino; falta de professionais qualificados, temas que furam expostos repetidamente nas mensagens emitidas pelo governo. 
Palavras chave: Revista história da educação latinoamericana; descentralização do ensino; instrução pública; história da educação em Mato Grosso.

\section{PIKÜNOGETUN}

Tüfa chi chijka mew ta malügeay ñi tukunewengen kimeltuwün zugu mato-grossense mew 1889-1908 xipantu, günezuamgen mew ta chem norgünen zugu mew feypilu ta pu koneltulu chijka xipalu fey chi mew.

Fey mew ta azkünugey epu ramtun inayael ta günezuam zugu: ¿müley tutewün zugu igkan chijkatuwe ruka pública moderna egu chumgen ta chijkatuwe ruka mülekefel Cuiabá, capital del estado Mato Grosso mew?; ka ¿chijkatuwe ruka pública cuiabanas elugepeygün fijem $\mathrm{ka}$ küme ruka kimeltuam kiñe wejin mew?. Tüfachi zugu nentugey kuyfike zugu mew chempin ta presidentes estatales, norgünen zugu mew kam decretos koneltulelu ta pública kimeltuwün ka fey tüfey chi chijka zugu xipalu 1889-1908 xipantu mew. Zewman mew tüfa chi küzaw kimgey ta 1889 xipantu mew, fey chi xipantu anümgelu República de Brasil, mülekefuy zewma ta wezake zugu chijkatuwe ruka Mato Grosso mew, müley warun, gelay chemkün kimeltucheam, ka gelay kümeke kimeltuchefe, fey chi ke zugu afkentu feypi ta pu günenielu fey chi mew.

Zichul zugun: Revista historia de la educación latinoamericana; descentralización de la educación; instrucción pública; chumlefuy kuyfi kimeltuwün Mato Grosso mew.

\section{INTRODUCCIÓN}

El trabajo investigativo surge de la necesidad de responder a las preguntas en torno a la consolidación de la educación pública en el estado de Mato Grosso (Brasil), debido a que los pocos estudios existentes no permiten detallar cómo se construyó y cuáles fueron los factores que permitieron su afianzamiento después de la descentralización de la educación, promulgada en la Constitución Federal de 1901. Por lo tanto, a través de un profundo examen de los archivos históricos de la ciudad de Cuiabá (capital mato-grossense) fue posible recolectar suficiente información histórica que posibilitara el desarrollo del proyecto. Así, el objetivo principal de este artículo es mostrar cómo se consolidó la educación pública en Mato Grosso, teniendo en cuenta los movimientos políticos que se generaban en el país.

En los documentos revisados, pertenecientes a los comienzos de la época republicana de Brasil, encontramos que la falta de estructura del proyecto educativo en el estado de Mato Grosso obligó a replantearlo por completo. Pudimos visualizar que, para la época, la infraestructura era casi inexistente y era generalizada la precariedad de las instituciones educativas, además de que, al no contar con autonomía municipal, no era posible investigar las iniciativas locales en la educación pública; por lo tanto, fue necesario extender la discusión para entender y explicar cómo se había llevado a cabo la descentralización del funcionamiento de la educación pública en el estado de Mato Grosso.

Para soportar el análisis tuvimos que detenernos en algunos textos sobre la Instrucción Pública en Mato Grosso, entre ellos la obra Um século de instrução 
pública: história do ensino primário em Mato Grosso ${ }^{4}$, en la cual se hace un breve recorrido por los cien años de educación pública que en 1970 cumplía el estado. Así mismo, el material producido por Humberto Marcílio ayudó a rever la historia de la enseñanza en Mato Grosso en el periodo de estudio. Cabe destacar que ambos autores son considerados memorialistas, es decir, autores encargados de escribir las memorias históricas de la educación mato-grossense.

Otro aporte esencial para entender la descentralización de la educación pública en Mato Grosso es el de Sandra Jung de Mattos, quien analizó el proceso histórico sobre la organización de la educación femenina en los inicios del periodo republicano (1889-1910). La autora mapea las escuelas femeninas destinadas a la enseñanza primaria en el estado de Mato Grosso, discutiendo las leyes, decretos de la Instrucción Pública y las normas para la escolarización femenina, con el objetivo de responder cómo se organizaba la educación femenina en términos de cuáles, cuántas y dónde estaban ubicadas las escuelas para mujeres en Mato Grosso entre 1889 y 1910. Jung de Mattos constató que la república brasileña propuso cambios para la instrucción pública en Mato Grosso, configurando diferencias notorias para la educación femenina, perpetuando valores que le atribuían a la mujer la misión de administradoras y cuidadoras del hogar. Además de ello, expone la autora, eran tan pocas las escuelas femeninas que las mujeres difícilmente podían acceder a la educación ${ }^{5}$.

Nos quedaba claro entonces que debíamos tener en cuenta ciertos antecedentes que eran esenciales para profundizar en la comprensión de la historia de las escuelas y la educación pública en el contexto específico del estado de Mato Grosso, situado en la vasta región brasileña denominada Centro-Oeste. Por ello, inicialmente buscamos comprender cómo el gobierno local trabajó en la consolidación de la educación pública en el estado, en lo que respecta a los proyectos políticos, el desarrollo económico y para las inversiones en la escuela. De allí que el corte cronológico propuesto inicia en 1889, cuando en Mato Grosso la Reforma de Souza Badeira instituyó nuevas directrices pedagógicas que determinaron grandes cambios en la estructura administrativa. Los años posteriores a la instalación de la reforma, hasta 1908, constituyen una referencia importante porque trajeron una serie de reglamentos sobre la instrucción que fueron emitidos con la intención de que "la acción del tiempo y la experiencia de las luces constituyeran la indicación de las reformas que deben operar a fin de refundir de una vez la ley oficial" ${ }^{\prime}$.

La propuesta de finalizar el estudio en 1908 se debió a los cambios efectuados en el objeto propio de la investigación, puesto que el 16 de octubre de ese año el gobernador del estado, Pedro Celestino Correa da Costa, bajo la resolución n. ${ }^{\circ}$ 508 , estableció varias escuelas primarias, autorizó la creación de los grupos de

4 Gervásio Leite, um século de instrução pública: história do ensino primário em Mato Grosso (Goiânia: Editora Rio Bonito, 1970).

5 Sandra Jung de Mattos. "Trabalhos de agulha e prendas domésticas: educação feminina mato-grossense (1889-1910)" (tesis de maestría en educación, Universidade Federal de Mato Grosso, 2018).

6 Manoel José Murtinho, "Mensagem do Presidente do Estado de Mato Grosso à Assembleia Legislativa" (Cuiabá: Typhografia do Estado, 1894). [Nuestra traducción]. 
escuela e inició un cambio en la estructura operativa de las instituciones educativas con un amplio proceso de nucleación. También fue el responsable de la extinción de algunas escuelas y la ampliación de otras, incorporando estudiantes y profesores de los establecimientos extintos, lo que se conoció en Mato Grosso, como la "Revolución de la Educación"7.

Por otra parte, identificamos que en 1908 se inauguró una nueva etapa para la educación pública y a partir de allí logramos construir una nueva comprensión sobre el objeto de estudio y, por ende, de la acción del Estado en relación con la educación que comenzó a construirse en Mato Grosso, influenciada, por supuesto, en ese momento histórico, por la actuación republicana en búsqueda de grandes iniciativas para el sector educativo. Estas acciones, aunque esparcidas por todo el país desde los años anteriores, solo se hacen visibles en Mato Grosso a partir de 1909.

\section{Los inicios de la educación pública en Mato Grosso}

Manoel José Murtinho, presidente de la provincia de Mato Grosso en 1889 y luego presidente del estado de Mato Grosso entre 1891 y 1895, al referirse a la educación pública hace hincapié en que era necesario que el Estado siguiera con la organización de los servicios que le incumben, en clara alusión a lo poco que este había hecho con relación a ella ${ }^{8}$. Según Murtinho, esta situación se debía a la escasez por la que pasaba el estado y la incapacidad para proporcionar los recursos necesarios para el desarrollo y el mantenimiento escolar, inclusive de dotar a los municipios de la infraestructura y los materiales didácticos necesarios para las clases.

En la lectura y análisis de los mensajes de quienes ocuparon la presidencia del estado durante el período estudiado, encontramos la preocupación explícita por la educación pública en Mato Grosso, aunque también resaltan el desarrollo del estado y las dificultades de acceso a las localidades, así como el escaso presupuesto del gobierno y los municipios para atender a una población tan dispersa, en especial en cuanto a la implementación de la educación pública gratuita y obligatoria. En una comunicación fechada en el mes de mayo de 1893, el Dr. Manoel José Murtinho, informaba a la Asamblea Legislativa:

Hasta la fecha todavía no se puede comenzar a poner en práctica el principio de la enseñanza primaria obligatoria, consagrado en la Constitución del Estado y lo establecido en el Reglamento. Con una población tan dispersa, como la que tenemos, es muy doloroso, si no imposible, en muchos lugares, la asistencia regular a las escuelas públicas para niños que necesitan educación y en tales condiciones, la obligatoriedad se convierte en esta forma de iniquidad ${ }^{9}$.

7 Humberto Marcílio, História do ensino em Mato Grosso (Cuiabá: SECS, 1963).

8 Murtinho, "Mensagem".

9 Ibíd. 
De manera que ya corría el año de 1893 y el presidente del estado había hecho poco o nada para cumplir con la obligatoriedad de la educación pública, a pesar de las diversas leyes, decretos y reglamentos estipulados para tal fin. La regulación establecida el 28 de mayo 1889, el Decreto n. ${ }^{\circ} 10$ del 7 de noviembre 1891, el Decreto n. ${ }^{\circ} 68$ del 20 de junio de 1896, la Ley n. ${ }^{\circ} 152$ del 16 de abril de 1896 y el Decreto n. ${ }^{\circ} 139$ del 2 de enero de 1903, en lo que se refieren a la educación en Mato Grosso, intentaron esbozar un "plan de educación" con la presencia de una legislación descentralizadora de la gestión educativa que se correspondiera con el propósito de una educación que atendiera a la población de Mato Grosso.

La causa principal para el incumplimiento de la obligatoriedad de la educación primaria era, evidentemente, la población dispersa a todo lo largo y ancho del estado de Mato Grosso. El mismo autor Murtinho afirma que en 1893 sólo había 62 escuelas públicas, 57 de ellas escuelas primarias o de primer grado. Así mismo, existían otras cinco escuelas de segundo grado, de acuerdo con la Ley n. ${ }^{\circ} 152$ del 16 de abril de 1896, que continuaban con la instrucción pública de la misma manera que en años anteriores. Muchos habitantes eran analfabetos y las escuelas eran poco frecuentadas. El estado tenía solamente una escuela secundaria y no había escuelas para la educación superior ${ }^{10}$.

Con una superficie de 1477041 kilómetros, Mato Grosso tenía una población de solo ochenta mil habitantes. Gervasio Leite afirma que la población dispersa y por ende sin educación, se distribuía en diez municipios aislados, en granjas y establecimientos de pastoreo con pocos caminos, dificultad en la recepción y transmisión de noticias y una mayor densidad de personas concentrada en Cuiabá. La educación en Mato Grosso estaba bajo la responsabilidad de un puñado de maestros. Este autor describe que la situación de la educación en el inicio de la República de Brasil en el suelo mato-grossense era difícil, las escuelas y los estudiantes eran casi inexistentes ${ }^{11}$.

Tabla 1. Escuelas y alumnos mato-grossenses en los inicios de la república

\begin{tabular}{|l|l|}
\hline Escuelas primarias del sexo masculino & 20 \\
\hline Escuelas primarias del sexo femenino & 12 \\
\hline Total & 32 \\
\hline Alumnos matriculados del sexo masculino & 963 \\
\hline Alumnos matriculados del sexo femenino & 516 \\
\hline Total & 1479 \\
\hline
\end{tabular}

Fuente: Leite, Um século de instrução pública.

En la tabla 1 es posible analizar varios aspectos. En primer lugar, la notable diferencia entre el número de escuelas masculinas y femeninas lo cual, seguramente, influía directamente en el segundo aspecto: los niños matriculados

10 Ibíd.

11 Leite, Um século. 
casi doblan al número de niñas. Con estas diferenciaciones, es de considerar lo que generaría para la nueva República esta brecha entre la educación pública de hombres y mujeres, sin duda un mayor índice de analfabetismo en el sexo femenino.

Ahora bien, en términos generales, teniendo en cuenta que la comunidad mato-grossense para la fecha estaba conformada por ochenta mil habitantes, se puede inferir que la población matriculada en las escuelas era escasa, puesto que no representaba ni el dos por ciento del total de habitantes, debido, como ya se dijo, a la dispersión de los pobladores y las dificultades de comunicación, sumado a ello, la escasez de recursos.

En los primeros tiempos del nuevo régimen republicano se produjo una gran confusión en la provincia brasileña de Mato Grosso que trajo como resultado numerosos cambios políticos y educativos y, con ello, problemáticas que los ciudadanos y los gobiernos tuvieron que asimilar. Los primeros cambios promovidos por la República de Brasil fueron la introducción de la escuela sin Dios, la educación laica y gratuita y el formalismo de la enseñanza obligatoria. El requisito de obligatoriedad educativa, que, si bien tenía como propósito acercar a todos a la educación pública, hizo surgir una nueva necesidad presupuestaria que estados y municipios no podían cubrir. El gobierno no contaba con una previsión de nuevas fuentes de ingresos para los estados y municipios, teniendo en cuenta que, en 1889, los ingresos de Mato Grosso fueron de R $\$ 215905143^{12}$; y los gastos de $\mathrm{R} \$ 204815203^{13}$, sin prever en ellos el costo de la educación.

Sumado a la necesidad urgente de un presupuesto destinado para la educación pública, la incertidumbre política y económica causada por las luchas internas por el poder entre políticos y gobernantes rivales crearon un ambiente inapropiado para las discusiones relacionadas con la educación oficial. Los problemas más importantes eran de orden político y consistían en salvaguardar y consolidar la República de Brasil, dejando de lado la crisis educativa que se estaba produciendo.

\section{El movimiento de descentralización de la educación en Mato Grosso}

Apenas dos años después del advenimiento de la República del Brasil el 15 de noviembre de 1889-, se consolida, en 1891, la Constitución Federal del estado de Mato Grosso, el mismo año en que se proclamó la Constitución Federal de Brasil.

En cuanto a los principios de la descentralización de la educación pública del gobierno federal para los estados y municipios brasileños, encontramos que el Congreso Constituyente debatió el proyecto de Constitución Federal de Brasil elaborado por un comité compuesto especialmente para este propósito y nombrado por el gobierno provisional, que finalmente resultó en la Constitución de

12 En estas expensas no se evidencian presupuestos destinados a la instrucción pública, por lo que es necesario investigar e identificar fuentes que comprueben cómo la instrucción pública subsistía en la época y a cuáles recursos acudía para su manutención.

13 Los valores se dan en la moneda brasileña del periodo, denominada réis. 
1891 como presidencial, democrática y liberal; sin embargo, esta Constitución casi nada preveía para la educación o la forma en que debía organizarse en los estados y municipios ${ }^{14}$.

Carlos Roberto Jamil Cury afirma que el proceso constitucional, en lo atinente al tema de la educación, se tradujo en tres temas. El primero se refiere a la organización, a la esfera pública dividida en términos de la Unión y las unidades federales, a la libertad de apertura de las escuelas en el mercado, lo que resultó en la división de competencias: la educación pública primaria fue delegada a los estados y municipios; la enseñanza secundaria fue declarada responsabilidad de los estados, pero también podría ser mantenida por la Unión y, en contraposición, por el sector privado. La educación superior fue considerada privada en la Unión, abierta a los estados y libre a la empresa privada. El segundo tema se refiere al laicismo (la separación del Estado de la Iglesia), lo que determinaba una posición no-religiosa en las escuelas públicas. Por último, el tema de la educación obligatoria y gratuita de la enseñanza primaria, con el cual se evidencia una omisión puesto que ni una cosa ni la otra fueron responsabilidad de la Unión. El asunto de una instrucción gratuita causaba numerosos problemas en la adecuada implementación de la educación pública en los pueblos, ya que no se contaba con disposición presupuestaria en ninguna de las agencias estatales: federal, estatal o municipal, para el mantenimiento ${ }^{15}$.

Para Ana María Ferreira Menezes, en 1889, la república, fundada en el principio del federalismo, con la creación de las legislaciones estatales y municipales, se había consolidado a través de un arreglo institucional basado en un sistema con más de un nivel de autoridad, cada uno con la responsabilidad de tomar decisiones independientes ${ }^{16}$. Consideramos que este hecho no daría lugar a una consolidación de un sistema de educación pública eficaz, ya que los estados y los municipios terminaron relegando la educación de la población, una vez se consideraban prioritarias otras acciones.

Por su parte, la Constitución de 1891, en su Art. 34, le otorgó, por tanto, a la Unión, la competencia de la organización de la educación superior y el Distrito Federal, lo que deja, consecuentemente, la enseñanza primaria y secundaria a los estados y a los municipios. En el informe presentado en 1891, encontramos que:

En los meses que siguieron a la proclamación de la República, la administración general, por lo menos en lo que concierne al Ministerio a mi cargo, sufrió un ligero

14 Messias Costa, A educação nas Constituições do Brasil: dados e direções (Rio de Janeiro: DP \& A Editora, 2002); Justo Cuño Bonito. "La Universidad Latinoamericana en la Encrucijada: Amenazas, Desafíos y Soluciones" Revista Historia de la Educación Latinoamericana Vol. 18, no. 26, (2016): 241-77. https://doi.org/10.19053/01227238.4374.

15 Carlos Roberto Jamil Cury, Cidadania republicana e educação: Governo Provisório do Mal. Deodoro e Congresso Constituinte de 1890-1891 (Rio de Janeiro: DP \& A Editora, 2001).

16 Ana Maria Ferreira Menezes, "A autonomia e os recursos tributários municipais no Brasil: uma análise à luz das Constituições Republicanas", Bahia Análise \& Dados 13, no. 3 (2003): 802. 
movimiento de la descentralización. Los primeros actos tuvieron como elemento inspirador la idea de conceder la autonomía inmediata a los estados ${ }^{17}$.

Los autores mencionados parecen coincidir en que, tras la proclamación de la república, en 1889, se incumplió la promesa republicana de una mayor democratización de la sociedad brasileña y su efecto retroactivo en la educación pública que debía ofrecérsele a la población. Por lo tanto, la forma en la que el régimen republicano se constituyó en la República de Brasil posibilitó al liberalismo fusionarse a la cultura política de un país colonizado, contrarreformista, esclavista y jerárquico, lo que daría como resultado profundas contradicciones en los distintos sectores de la élite en cuanto a la concepción y la necesidad de democratización de la escuela que atendiera al pueblo brasileño ${ }^{18}$.

Para Gonçalves Neto, las mismas características del Imperio del Brasil se reprodujeron en la República ${ }^{19}$. Se mantuvieron las correlaciones de analfabetismo, la ignorancia y el atraso económico, incluso con el discurso de la descentralización de las decisiones políticas con implicaciones en las localidades. En esta perspectiva, la Constitución Federal comprometía a que cada estado asumiera su organización, de modo que garantizaba la autonomía de los municipios en todo lo concerniente a su interés particular ${ }^{20}$. Así que se determinó que la descentralización de la educación pública debería tener un sentido mucho más burocrático que político.

Es posible inferir que las discusiones entre las élites políticas e intelectuales, en los primeros años de la etapa republicana, se mantuvieron fuertemente centralizadas. Los estados y los municipios no podían establecer un enfoque coherente para actuar con eficacia en el desarrollo de programas de estudio, la actualización de los métodos y técnicas de enseñanza. Ellos mismos presentaban dificultades para proporcionar formación al profesorado, adquirir insumos pedagógicos, libros de texto, proveer una organización de los espacios de tiempo y de la escuela, crear y proporcionar el mantenimiento de las escuelas. Hecho que se repitió en todos los estados de la nueva república, incluyendo Mato Grosso y sus municipios.

Ahora bien, con la Constitución del estado de Mato Grosso, promulgada en 1891, los obstáculos que enfrentaría el buen funcionamiento de las escuelas públicas no fueron previstos o explicados. Esta primera Constitución del estado de Mato Grosso instituyó, sin embargo, principios de descentralización. En el primer título, Organización Estatal, predijo que el gobierno de la Unión no haría

17 Presidencia de la República, Constituição da República dos Estados Unidos do Brasil (Brasil, Presidencia de la República, 1891). [Nuestra traducción].

18 Rosângela Mendonça Teles, "O Papel dos Conselhos de Educação na Formação dos sistemas Educacionais Brasileiros”, en la Reunião Anual da Associação Nacional de Pós-graduação e Pesquisa em Educação (ANPED, 2001).

19 Wenceslau Gonçalves Neto, "O Município e a Educação em Minas Gerais: a implementação da Instrução Pública no início do Período Republicano”, en O Município e a Educação no Brasil: Minas Gerais na Primeira República, eds. Wenceslau Gonçalves Neto y Carlos Henrique de Carvalho (Campinas: Alínea, 2012), 28.

20 João Barbalho Uchôa Cavalcanti, Relatório apresentado ao Presidente da República dos Estados Unidos do Brazil em maio de 1891 (Rio de Janeiro: Imprensa Nacional, 1891). 
ninguna intervención en el estado distinta de las previstas por la ley. En el segundo capítulo, acerca de los Poderes de la Legislatura, señaló que la educación pública sería libre y laica en todos sus grados, así como la instrucción primaria sería gratuita y obligatoria, acatando las condiciones establecidas por la ley (Art. 11); en este mismo título se estableció que el municipio sería autónomo, independiente en sus negocios, y agregó que el municipio debía crear, mantener y subvencionar las escuelas de enseñanza primaria (Art. 52, § 16).

En este sentido, podemos afirmar que, en el camino de la democratización de la sociedad y el desarrollo, las constituciones republicanas de Brasil y en específico del estado de Mato Grosso, propusieron que los municipios jugarían un papel preponderante. Esto quiere decir que no debían representar la mera función de apoyo o complemento de la función del Estado, sino que los municipios debían actuar de forma autónoma en los servicios y los bienes de los que carecían los ciudadanos, incluyendo así la educación pública.

\section{Municipio pedagógico y descentralización: el estudio de la localidad}

En este marco multifacético, el estudio de lo local adquirió especial importancia para comprender la forma en la que los municipios de Mato Grosso organizaron la educación pública y, por otro lado, entender cómo el Estado y los municipios compartían perspectivas similares a las que guiaban el debate educativo brasileño. Para posibilitar una mirada más amplia sobre la actuación de las localidades como promotoras de la educación pública, creemos necesario reflexionar sobre lo que se designa como local. En el plano histórico, la respuesta a esa pregunta implica una dialéctica entre los conceptos: municipios pedagógicos y descentralización.

El municipio pedagógico, de reciente divulgación, se refiere al reconocimiento de un caso particular o a una cierta potencia necesaria para mantenerse a sí mismos, de autonomía. En ese sentido entendemos, como lo expone Gonçalves Neto, que el municipio pedagógico es una categoría en construcción ${ }^{21}$; una entidad político-administrativa que se hizo presente en Brasil desde finales del siglo XIX por la presencia de una ley descentralizadora de la gestión de la enseñanza y la extensión de la responsabilidad de la educación primaria a sus límites, lo que lleva a la regulación local de esta obligación.

Otro autor portugués, Justino de Magalhães, señaló que el municipio político y administrativo siempre envolvió la construcción del municipio pedagógico ${ }^{22}$. Es decir, en los períodos históricos de mayor afirmación de los países, al ser los municipios una entidad importante en el marco político y administrativo del país, se encargaron de desarrollar estrategias de integración y de valoración local a través de la educación pública.

21 Gonçalves Neto, "O Município e a Eeducação".

22 Justino Magalhães, “O local e a decisão política em educação: aspectos teóricos e metodológicos da história do Município Pedagógico”, en Actas do VII Congresso Luso-Brasileiro de História da Educação (Portugal: CLBHE, 2008). 
Retomando a Gonçalves Neto, el municipio pedagógico constituye, entonces, "un poder local con la capacidad de tomar iniciativas políticas, discutir y aprobar leyes, en complemento o más allá de las facultades concedidas por los estados"23. Por ello, la investigación tomó como principio rector la idea de que el estado de Mato Grosso hubiese propiciado en las localidades el surgimiento de los sistemas educativos municipales, principalmente debido a la omisión o el fracaso de los esfuerzos estatales para proveer la instrucción pública obligatoria y gratuita a la población, según lo establecido por la Constitución del estado; en Mato Grosso los gobernantes privilegiaron la necesidad de buscar soluciones a los problemas de la vida social, política y económica.

Una de las primeras iniciativas para la organizar la instrucción pública en Mato Grosso después de la proclamación de la república surgió con el Decreto n. ${ }^{\circ} 10$ del 7 de noviembre de 1891. En este decreto se estipula, en su capítulo 12, un fondo escolar para el mantenimiento de las escuelas.

\begin{abstract}
Artículo 86 - Se crea un Fondo Escolar, que tiene como fuente principal un impuesto de capitación a la razón de dos mil reales por cada contribuyente anualmente en las ciudades que fuesen cabeceras municipales y mil reales en otras ciudades $y$ villas.

$\S 1$ - Este impuesto será cargado en todas las personas que viven en el estado, que ejercen la industria, profesión o viven de ingresos propios. § 2 - La liquidación y recaudación de este impuesto se hará de conformidad con los artículos 29 a 35 del reglamento provincial del 22 de abril 1889.

Artículo 87 - El Fondo Escolar estará constituido también: I - De las cuotas pagadas por razón de la designación, remoción, la gratuidad, permiso o sustitución de los profesores y empleados de la Instrucción Pública. II - emolumentos recibidos en virtud de los certificados [fl.33v] aprobadas por la Secretaría de la Instrucción Pública. III - El producto de las multas impuestas en el presente Reglamento. IV - De las donaciones o legados hechos a la tesorería del Estado a favor de la instrucción pública.

Artículo 88 - El Fondo Escolar se destinará al desarrollo de la instrucción pública, aplicándose preferentemente a la adquisición de mobiliario para las escuelas, y la provisión de ropa y utensilios a los niños indigentes de conformidad con el artículo 85.24
\end{abstract}

El Decreto n. ${ }^{\circ} 10$ de 1891 incorpora varios elementos esenciales para la educación pública de Mato Grosso, como lo son la creación de un fondo para las escuelas, el cual debía ser utilizado para dotar a las instituciones de mobiliarios necesarios y de materiales de estudio para los niños que no estaban en condiciones de adquirirlos. Este fondo, si bien no provenía directamente del presupuesto del estado sino de los impuestos, multas y demás acciones ciudadanas, consti-

23 Gonçalves Neto, "O Município e a Educação". [Nuestra traducción].

24 Manoel José Murtinho, Governo de Mato Grosso, Decreto no. 10 de 07 de novembro (Cuiabá: Typhografia do Estado, 1891). [Nuestra traducción]. 
tuía una forma de ofrecer a las escuelas la posibilidad de adquirir el dinero para intentar su sostenimiento.

Sin embargo, las regulaciones establecidas por el Decreto n. ${ }^{\circ} 10$ de 1891 acabarían por no proporcionar los recursos necesarios para la educación pública en el periodo, ni por ampliar el servicio de la población en lo que respecta a la educación popular, lo cual se evidencia en el mensaje de Murtinho en 1893, cuando menciona que albergaba la esperanza de que ese año se pudiera ejecutar la reglamentación expresada por la Constitución, pero advierte sobre las numerosas dificultades que impiden que se cumpla.

Pero otra condición depende también de la exigencia de la educación, pues para que los niños asistan a la escuela es indispensable proporcionar ropa $y$ utensilios a quienes son indigentes, cuyos gastos deberían correr por cuenta del fondo escolar. Este fondo aún está por conformarse, puesto que el impuesto de capitación, que es la principal fuente de ingresos creada [...] dejó de ser elevado hasta ahora, si bien yo ya haya hecho arreglos para su colección ${ }^{25}$.

Con el fondo escolar, creado por Murtinho en 1893 como fuente de ingreso para apoyar económicamente a las escuelas del estado mato-grossense, se esperaba que los beneficios líquidos de las loterías del estado se aplicaran en la educación pública, con el fin de evitar su extracción del impuesto general, lo que reducía considerablemente los recursos asignados a la educación, aliviando el seguro estatal. No obstante, señaló que estos recursos requerirían "no pocos años" ${ }^{26}$, pero que representarían un valioso subsidio a la educación pública en el estado. Por lo tanto, justifica el retraso de la puesta en práctica de la educación pública gratuita y obligatoria y posterga su implementación mientras llegaban los recursos necesarios para costearla.

En el mismo mensaje de 1893, Murtinho declaró que no sería posible elevar el pago de los salarios del profesorado para poder cumplir con la educación pública obligatoria, ni dotarlo de los equipos necesarios para el buen funcionamiento de las clases, incluyendo las comodidades propias necesarias.

De visita en las escuelas públicas primaria de esta capital tuve la oportunidad de comprobar que, a excepción de las dos que ocupan edificios estatales y disponen de comodidades deseables, todas las más se encuentran mal instaladas en casas de alquiler, funcionando las clases en habitaciones insuficientes, y algunas, incluso, sin las más elementales condiciones higiénicas ${ }^{27}$.

Es de apreciar que Murtinho afirmaba que la falta de recursos económicos destinados a las escuelas públicas del estado no solo afectaba directamente a la infraestructura de sus instalaciones, sino también el pago oportuno a los docen-

25 Murtinho, "Mensagem". [Nuestra traducción].

26 Ibíd.

27 Ibíd. 
tes. De ello se deduce que las grietas creadas en la educación pública fueron tan sustanciales que aún podrían estar vigentes.

Además de ello, Murtinho señaló que existía una falta de edificios propios para la instalación de las escuelas, sugiriendo así la necesidad urgente de construir edificios apropiados para "señores de la enseñanza" ${ }^{28}$, lo cual estaba a tono con las discusiones sobre los temas educativos que se habían intensificado con el establecimiento de la república. Incluso, Murtinho llegó a elaborar un anteproyecto sobre la construcción de un edificio de escuela, pero ello no garantizó la adopción de propuestas coherentes para la educación obligatoria, puesto que la razón principal del incumplimiento de lo estipulado por la ley, empezando por la falta de estructura física, fue la no disposición de recursos presupuestarios.

Tengo previsto enviar a fabricar en la dirección de obras públicas la planta de un edificio con los requisitos necesarios para los quehaceres escolares, modesto edificio, bien sea elegante, y de un costo relativamente módico, de modo que en cada ejercicio pueda hacerse efectiva la construcción de una o más casas de aquel tipo, que correspondan a los recursos monetarios para proporcionar el presupuesto ${ }^{29}$.

De la cita anterior se desprende que se requería una innovación en la educación. Los espacios destinados para las escuelas públicas debían ser mejorados. La discusión planteada sobre los edificios y las condiciones de higiene exigía nuevos ajustes para la educación pública y nuevas construcciones, aunque modestas, para iniciar un nuevo sistema de educación pública proporcionado por el presupuesto que se derivaba de las loterías. Con todo, cabe señalar que esa aplicación surge porque se había estipulado por la Unión cuando se distribuyó el recurso.

Sin embargo, para Murtinho parece innecesario aplicar el recurso en la educación pública ${ }^{30}$. Él explicó que debería ser suficiente para la exención de impuestos, y añadió ...que chegaria quasi a absorver os mesmos benefícios ${ }^{31}$. Con ello, hace referencia a la enseñanza de que "se practicaba" y las bajas tasas de finalización de los estudiantes detectado desde 1889, afirmando por tanto que no necesitarían más inversiones.

Extraída en la capital federal durante el año pasado, la importante suma de once o menos reis, los cuales tengo que gastar en la instrucción pública, de acuerdo con el compromiso que se contrajo con el ministro de Hacienda a fin de obtener la exención del impuesto general ${ }^{32}$.

31 “...habría llegado casi a absorber los mismos beneficios". [Nuestra traducción].

32 Murtinho, "Mensagem".
} 
Observamos que el mensaje de Murtinho ocurrió como un intento, aún incipiente, de ocupar una posición de toma de decisiones en el campo de la educación cuando se propuso entrar en un "sistema de construcción" 33 , confiando en los beneficios de la lotería. En esa decisión se encuentran las percepciones de la educación en el período, de un tiempo y de una propuesta educativa coherente con las concepciones modernas que la república intentaba instalar.

En esa posible acepción moderna, Murtinho se entrelazaba con el objetivo de aumentar los valores, concepciones, actitudes, modos específicos republicanos y, en especial, la producción y la reproducción de la organización de la vida política, por lo que necesitaba nuevas formas de almacenamiento financiero para el mantenimiento de la educación pública de Mato Grosso. Por lo tanto, constatamos que la educación obligatoria hasta 1893 no era más que un artículo dispuesto en la Constitución.

La política de moderación y justicia, seguida escrupulosamente por el gobierno, ha desarmado odios y contribuyó para operar la reconciliación de los ánimos [...] y ha permitido al Estado completar su evolución política y continuar con la organización de los servicios que le incumben ${ }^{34}$.

Persistían, pues, las disputas derivadas de la proclamación de la república. Por otro lado, comprobamos que los problemas existentes en las escuelas ubicadas en las ciudades continuaron: precariedad de la infraestructura; falta de recursos para la enseñanza; falta de profesionales cualificados, todo lo cual era continuamente expuesto en los comunicados de Murtinho, por lo que muchas escuelas no tenían incluso las condiciones mínimas de funcionamiento.

En el mensaje de 1894, el mismo presidente daba cuenta de que casi nada se había hecho por la educación pública. Recordó que ningún cambio se había llevado a cabo en el 1891 con la esperanza de que "la acción del tiempo y las luces de la experiencia den las indicaciones de las reformas que se deberán producir a fin de refundar de una vez por todas la legislación relativa a la educación pública" ${ }^{35}$.

También advirtió que "si bien ya se encuentra en el directorio del plan de obras públicas la planta para la construcción de un edificio modelo para las escuelas públicas, en esta capital no se le había mandado a construir uno de aquel tipo" ${ }^{\prime 36}$. Así mismo, expuso que esto se debía a la insolvencia por la que pasaba al estado y la incapacidad para pagar la mano de obra y los recursos necesarios para construir; sin embargo, afirmaba que se habían proporcionado algunas mejoras. Murtinho informó que los muebles y utensilios fueron proporcionados a las escuelas para "remediar" los problemas de las escuelas en la capital y algunas del interior.

\begin{tabular}{ll}
\hline 33 & Ibíd. \\
34 & Ibíd. \\
35 & Ibíd. \\
36 & Ibíd.
\end{tabular}


Como podemos apreciar en los mensajes presentados por Murtinho a la Asamblea Legislativa, el gobernante sostenía que, a pesar de la autorización legislativa para implementar las reformas educativas sugeridas, él no las había promovido por no considerarse cualificado con los datos que estaban disponibles o que sus directores habían recogido, para poner en funcionamiento la reforma que era necesaria. El reglamento de 1891 que prevalecía se tornó inoperante e ineficaz en el terreno de la educación pública mato-grossense. Fue solo hasta 1896 que una nueva regulación se instituyó. Esas dificultades identificadas en las escuelas ubicadas en la ciudad, cuya precariedad era continuamente manifestada en los informes de los presidentes estatales, nos llevó a identificar que las pocas escuelas existentes en el inicio de la república no habían tenido ni siquiera las condiciones mínimas de funcionamiento.

Diez años después de la proclamación de la república, el informe presentado por el director general de Instrucción Pública, Joaquim da Silva Rondon, al coronel Antonio Pedro Alves de Barros, presidente del estado de Mato Grosso, describe:

La lucha actual contra la falta de personal calificado para regir las escuelas establecidas, con la falta de viviendas adecuadas y útiles escolares indispensables debe limitarse a la acción de los directores de escuela, por ahora, para mejorar las condiciones de las escuelas existentes ${ }^{37}$.

Identificamos, en suma, total precariedad en la infraestructura escolar, falta de recursos para la enseñanza, escasez de profesionales cualificados, escuelas cuiabanas sin las condiciones mínimas de funcionamiento, con ausencia total de políticas públicas consistentes con las necesidades de la población cuiabana a ser escolarizada. Además de ello, poco interés de los gobiernos de la época en adoptar acciones sustanciales que permitieran mitigar las falencias por las cuales pasaba la educación pública de Mato Grosso.

\section{CONCLUSIÓN}

La necesidad del estudio de las relaciones políticas locales y de las articulaciones de las élites regionales en torno a la defensa de sus intereses, como en el caso de Mato Grosso, nos parece particularmente relevante para ponderar la educación brasileña en el contexto republicano. Hemos demostrado que la república había traído, al menos en los discursos de las autoridades públicas, la defensa de una escuela que podría promover la educación pública en las localidades; sin embargo, identificamos en la documentación analizada la ausencia de edificios y mobiliarios adecuados para un ambiente educativo, con materiales de

37 Joaquim da Silva Rondon, "Relatório apresentado ao Coronel Antônio Pedro Alves de Barros, Presidente do Estado de Mato Grosso, pelo Diretor Geral da Instrução Pública. Cuiabá, 30 de diciembre de 1899” (APMT, 1899). [Nuestra traducción]. 
enseñanza, profesores calificados y suficientes para la educación de la población mato-grossense.

Las dificultades encontradas en las escuelas ubicadas en los pueblos consistían en la precariedad verificada en infraestructura, la falta de recursos educativos, la falta de profesionales cualificados, lo cual se reflejaba constantemente en los informes oficiales. Las escuelas de Mato Grosso no poseían las condiciones mínimas de funcionamiento, como lo describen los presidentes. Para ellos, tan solo se pudiera tratar de mejorar las condiciones de las escuelas existentes y suficientes para satisfacer la educación popular.

Además de ello, consideramos que la divulgación de estos datos permite comprender el funcionamiento de la educación pública, su historia, sus obstáculos, sus contribuciones a nivel local, es decir, el papel que los municipios representaron y todavía representan en relación con la historia de la educación brasileña. Creemos que el estudio realizado sobre el estado de Mato Grosso en el inicio de la república puede añadir a la obra ya producida sobre la misma y contribuir a las nuevas miradas de las realidades: la existencia y la aparición del poder local con la capacidad de definir los principios y hacer leyes, intentando, al final, la creación de un "sistema" de educación en las municipalidades en el inicio de la república. Todo esto respaldado por una ley expandida en discusiones legislativas y en las cámaras municipales.

Por lo tanto, las fuentes que aquí exponemos no parecen servir solo para establecer los parámetros de la organización escolar en la educación pública oficial de Mato Grosso, sino también para comprender el choque de fuerzas en las relaciones político-administrativas que imponen un dispositivo a determinado sistema educativo, en un estado en el que prevalece la falta de políticas públicas para la educación popular.

A pesar de que la organización de la educación pública en Mato Grosso se menciona en los diversos informes de inspección y los diferentes mensajes de los presidentes, no se pudieron encontrar los medios para dar continuidad a un proyecto que alcanzara una educación que atendiera a la población -grossense en las localidades, como se indicaba en sus reglamentos y programas educativos.

\section{REFERENCIAS}

Cavalcanti, João Barbalho Uchoa. Relatório apresentado ao Presidente da República dos Estados Unidos do Brazil em maio de 1891. Rio de Janeiro: Imprensa Nacional, 1891.

Costa, Messias. A educação nas Constituições do Brasil: dados e direções. Rio de Janeiro: DP \& A Editora, 2002.

Cury, Carlos Roberto Jamil. Cidadania republicana e educação: Governo Provisório do Mal. Deodoro e Congresso Constituinte de 1890-1891. Rio de Janeiro: DP \& A Editora, 2001.

Cuño Bonito, Justo. "La Universidad Latinoamericana en la Encrucijada: Amenazas, Desafíos y Soluciones" Revista Historia de la Educación Latinoamericana Vol. 18, no. 26, (2016): 24177. https://doi.org/10.19053/01227238.4374.

Gonçalves Neto, Wenceslau. "O Município e a Educação em Minas Gerais: a implementação da instrução pública no início do período republicano". En O município e a educação no Brasil: 
Minas Gerais na Primeira República, editado por Wenceslau Gonçalves Neto y Carlos Henrique de Carvalho. Campinas: Alínea, 2012.

Leite, Gervásio. Um século de instrução pública: história do ensino primário em Grosso. Goiânia: Editora Rio Bonito, 1970.

Magalhães, Justino. “O local e a decisão política em educação: aspectos teóricos e metodológicos da história do Município Pedagógico". En Actas do VII Congresso Luso-Brasileiro de História da Educação. Portugal: CLBHE, 2008.

Marcílio, Humberto. História do ensino em Grosso. Cuiabá: SECS, 1963.

Mattos, Sandra Jung de. “Trabalhos de agulha e prendas domésticas: educação feminina -grossense (1889-1910)". Tesis de maestría en Educación. Universidade Federal de Mato Grosso, 2018.

Menezes, Ana Maria Ferreira. "A autonomia e os recursos tributários municipais no Brasil: uma análise à luz das Constituições Republicanas". Bahia Análise \& Dados 13, n. 3 (2003).

Murtinho, Manoel José. "Mensagem do Presidente do Estado de Mato Grosso à Assembleia Legislativa". Cuiabá: Typhografia do Estado, 1894.

Murtinho, Manoel José. Governo de Mato Grosso. Decreto n.ํำ 10 de 07 de novembro. Cuiabá: Typhografia do Estado, 1891.

Presidencia de la República. Constituição da República dos Estados Unidos do Brasil. Brasil: Presidencia de la República, 1891.

Rondon, Joaquim da Silva. "Relatório apresentado ao Coronel Antônio Pedro Alves de Barros, Presidente do Estado de Mato Grosso, pelo Diretor Geral da Instrução Pública. Cuiabá, 30 de diciembre de 1899". APMT, 1899.

Teles, Rosângela Mendonça. “O Papel dos Conselhos de Educação na Formação dos sistemas Educacionais Brasileiros". En Reunião Anual da Associação Nacional de Pós-graduação e Pesquisa em Educação. ANPED, 2001.

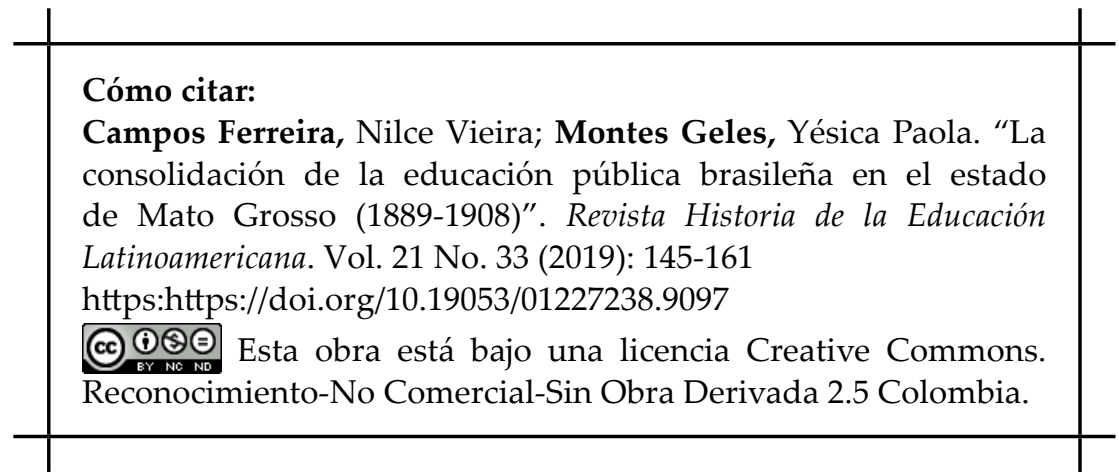

